\title{
CUT UMBILICAL CORD MILKING IS AS EFFECTIVE AS INTACT UMBILICAL CORD MILKING FOR HEMODYNAMIC ADAPTATION IN TERM INFANTS DURING TRANSITION PERIOD
}

Pamukkale University Faculty of Medicine Department of Paediatrics ${ }^{1}$, Division of Neonatology ${ }^{2}$, Department of Obstetric and Gynecology ${ }^{4}$, Denizli, Turkey ${ }^{4}$ State Hospital Department of Paediatrics Division of Neonatology, Denizli, Turkey

\section{Background and Aims}

Delayed cord clamping may not provide adequate placental transfusion in depressed infants and in neonates delivered by cesarean 1,2 . The aim of this study is to compare the effects of cut umbilical cord milking (C-UCM) and intact umbilical cord milking (I-UCM) which permit placental transfusion and resuscitation during transition period.

\section{Method}

Sixty-two healthy term infants not requiring respiratory support were randomized into C-UCM and I-UCM groups at birth. Approximately $25-30 \mathrm{~cm}$ length of cord was milked towards the baby 2-4 times within 20 seconds after birth in both C-UCM and I-UCM groups while the umbilical cord was cut in the former, and intact in the latter. Arterial oxygen saturation $\left(\mathrm{SpO}_{2}\right)$, cerebral regional oxygen saturation $\left(\mathrm{crSO}_{2}\right)$, heart rate $\left(2^{\text {nd }}-15^{\text {th }}\right.$ min), arterial blood pressure (within $15-30$ min), hemoglobin values (at the $6^{\text {th }}$ hour), and residual blood volume in the placenta (Fig. 1) were recorded during transition period after birth.

\section{Results}

There were no differences in terms of gestational age, birth weight, Apgar scores at $1^{\text {st }} / 5^{\text {th }}$ min, first respiration timing, mode of delivery, sex, blood pressures, residual blood volume in placenta, and hematologic parameters at 6th $\mathrm{h}$ (Table 1), SpO2, crSO2, and cFOE values between two groups except for HR ( $\mathrm{p}>0.05$ ) (Fig. 2). Mean HRs were significantly higher in C-UCM group at $6^{\text {th }}, 7^{\text {th }}, 9^{\text {th }}, 10^{\text {th }}, 12^{\text {th }}$, and $14^{\text {th }}$ min, but within normal range. HR and $c F O E$ levels of two groups were the highest, $\mathrm{SpO} 2$ and $\mathrm{crSO} 2$ levels were the lowest at $2^{\text {nd }} \mathrm{min}$, but within normal range. While HR decreased gradually, SpO2 increased continuously and reached $>90 \%$ at $7^{\text {th }} \mathrm{min}$, and $95 \%$ at $10^{\text {th }}$ min in C-UCM and I-UCM groups, respectively. The times to reach plateau level were found $10^{\text {th }}$ and $7^{\text {th }}$ min for SpO2, and $6^{\text {th }}$ and $6^{\text {th }}$ min for $\mathrm{CrSO} 2$ in C-UCM and I-UCM groups, respectively (Fig. 2)

\begin{tabular}{|c|c|c|c|c|c|}
\hline \multirow[t]{2}{*}{ Characteristics } & \multicolumn{2}{|c|}{ C-UCM $(n=31)$} & \multicolumn{2}{|c|}{ I-UCM (n=31) } & \multirow[t]{2}{*}{$\mathrm{p}$} \\
\hline & Mean $\pm S D$ & Med. (min-max), (n \%) & Mean $\pm S D$ & Med. (min-max), (n \%) & \\
\hline Gestational age (week) & $39 \pm 1.2$ & $39(37-41)$ & $38.8 \pm$ & $39(37-41)$ & 0.629 \\
\hline Birth weight (g) & $3351 \pm 254$ & $3300(2800-3800)$ & $3256 \pm 285$ & $3180(2860-3960)$ & 0.174 \\
\hline Cesarean delivery, $n$ (\%) & & $12(41.9)$ & & $13(45.2)$ & 0.769 \\
\hline Female sex, n (\%) & $17(54.8)$ & $14(45.2)$ & $16(51.6)$ & $15(48.4)$ & 0.799 \\
\hline First respiration timing (sec) & $5.42 \pm 3.87$ & $5(1-15)$ & $5.74 \pm 4.13$ & $5(1-15)$ & 0.848 \\
\hline Apgar score at $1^{\text {st }} \mathrm{min}$ & $8.8 \pm 0.3$ & $9(8-9)$ & $8.9 \pm 0.3$ & $9(8-9)$ & 0.691 \\
\hline Apgar score at $5^{\text {th }}$ min & $10 \pm 0$ & $10(10-10)$ & $10 \pm 0$ & $10(10-10)$ & 1 \\
\hline \multicolumn{6}{|l|}{ Blood pressure (mmHg) } \\
\hline Systolic & $74.3 \pm 8.1$ & $73(58-92)$ & $72.4 \pm 6.2$ & $72(60-89)$ & 0.3 \\
\hline Diastolic & $42.5 \pm 7.6$ & $41(31-58)$ & $42.1 \pm 7.4$ & $42(24-53)$ & 0.84 \\
\hline Mean arterial pressure & $52.9 \pm 6.9$ & $51(42-67)$ & $51.8 \pm 5.7$ & $53(39-61)$ & 0.86 \\
\hline \multicolumn{6}{|c|}{ Residual blood volume in placenta } \\
\hline $\mathrm{ml}$ & $80.16 \pm 18$ & $80(40-110)$ & $75.16 \pm 20.19$ & $75(45-135)$ & 0.308 \\
\hline $\mathrm{ml} / \mathrm{kg}$ & $23.96 \pm 4.76$ & $24.9(12.2-33.5)$ & $22.95 \pm 5.42$ & $22.4(15.3-40.4)$ & 0.439 \\
\hline \multicolumn{6}{|c|}{ Hematological parameters at $6^{\text {th }} \mathrm{h}$} \\
\hline $\mathrm{Hb}(\mathrm{g} / \mathrm{dl})$ & $17.7 \pm 1.8$ & $17.7(14.4-22.3)$ & $18.4 \pm 1.4$ & $18.6(15.6-22.7)$ & 0.111 \\
\hline $\mathrm{Htc}(\%)$ & $53.0 \pm 5.6$ & $53.5(44.8-65.5)$ & $55.3 \pm 4.4$ & $55(45.5-69.2)$ & 0.086 \\
\hline
\end{tabular}

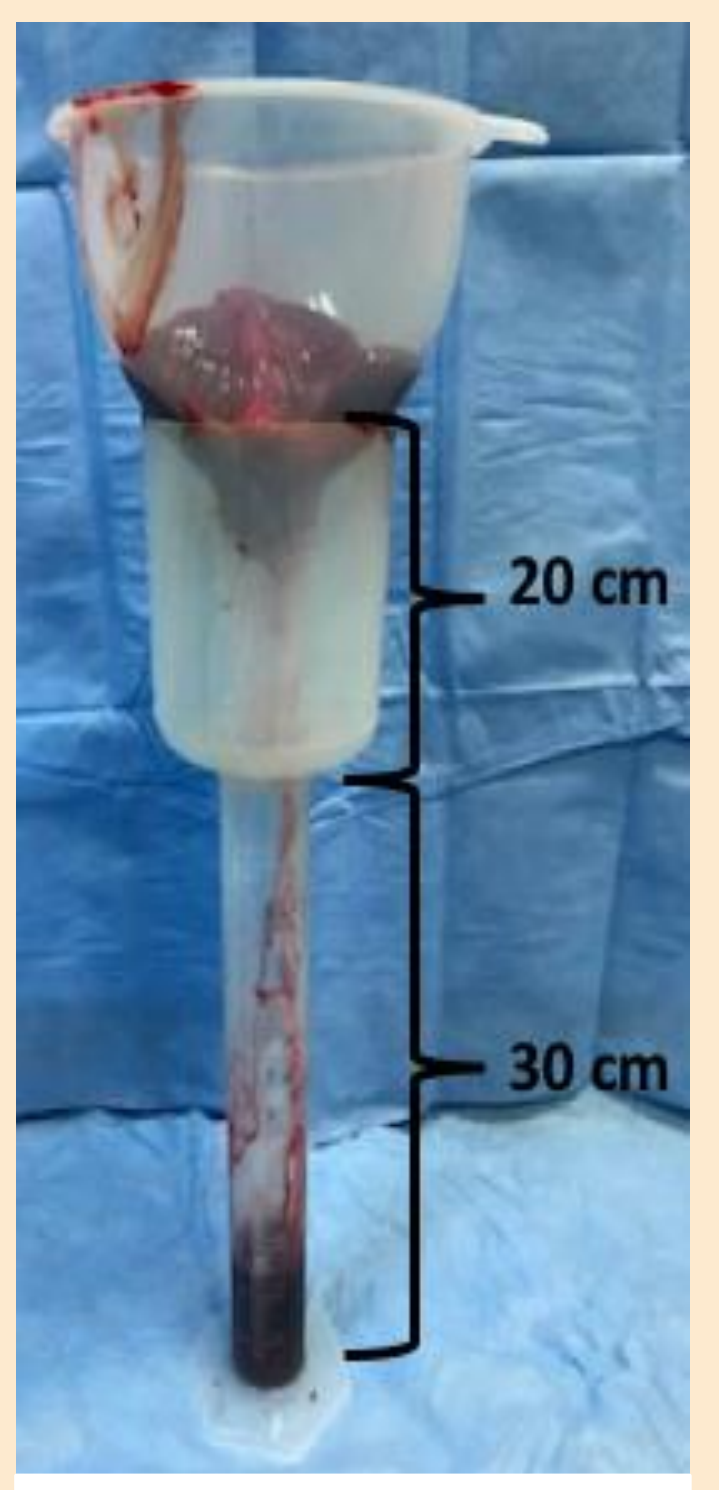

Fig. 1: Assembly prepared for measuring residual blood volume in placenta.

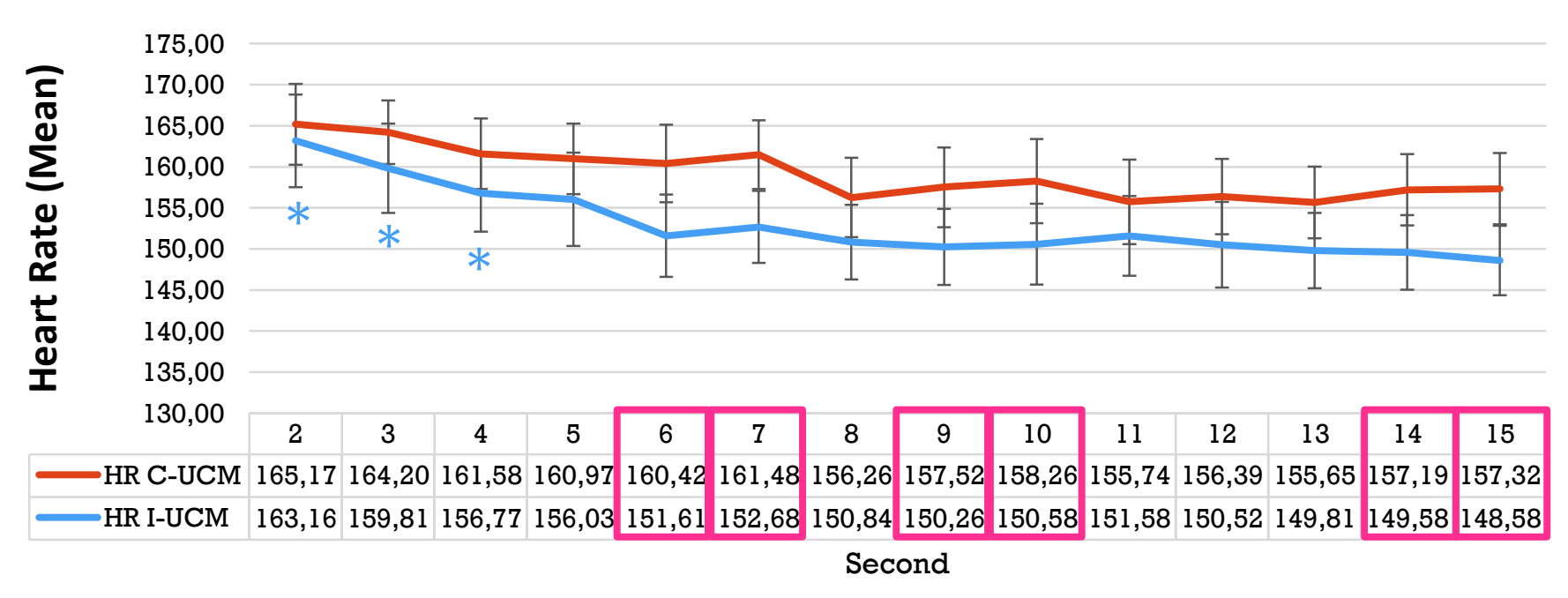

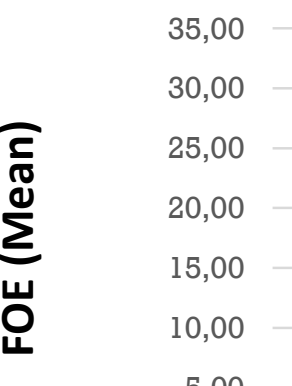
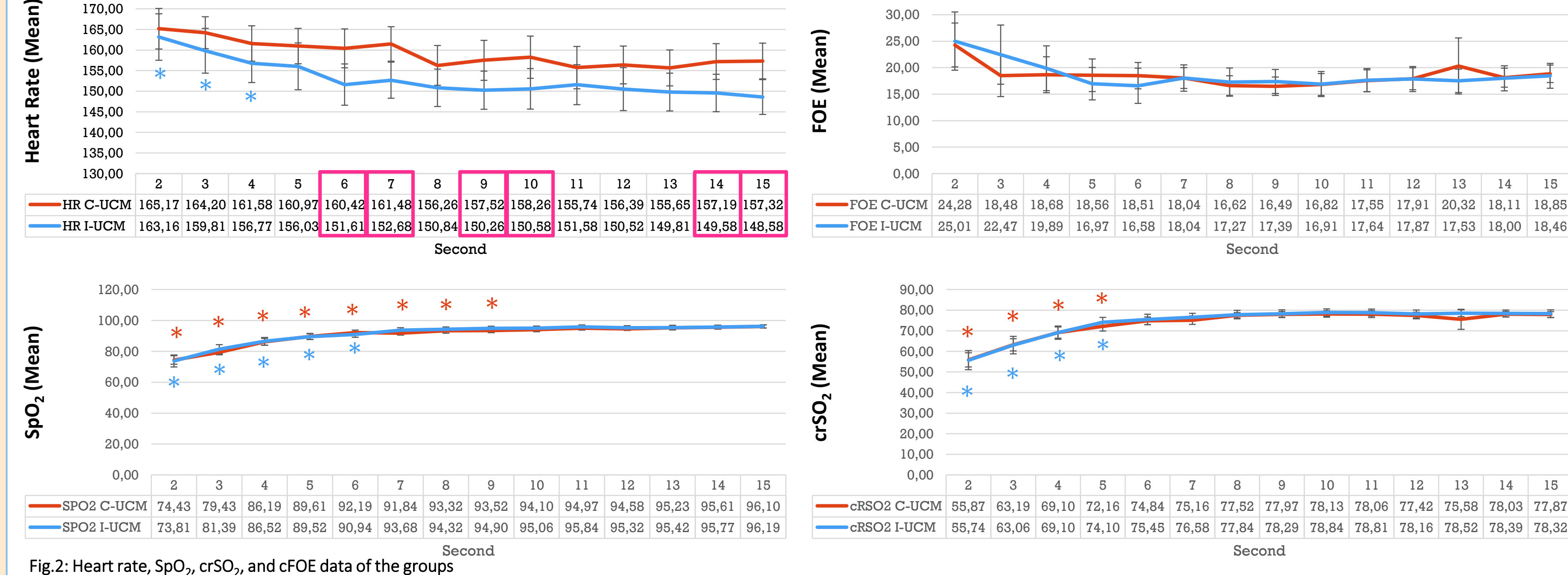

Conclusion This study showed that C-UCM is as effective as I-UCM at hemodynamic adaptation of healthy term infants during transition period. In light of these results, we suggest that C-UCM can provide placental transfusion in neonates requiring resuscitation and born by cesarean. 